\title{
Role of Finite Element Analysis in Oral and Maxillofacial Surgery - A Review
}

\author{
Manish Anand ${ }^{1}$, Shreya Panwar ${ }^{2}$, Srestha Bisht ${ }^{3}$ \\ 1,2 Department of Oral and Maxillofacial Surgery, Meenakshi Ammal Dental College, Chennai, Tamilnadu, India. \\ ${ }^{3}$ Department of Orthodontics, Subharti Dental College, Meerut, Uttar Pradesh, India.
}

\section{ABSTRACT}

\section{BACKGROUND}

Maxillofacial surgeries vary from simple tooth extraction to maxillofacial reconstruction and rehabilitation. The intricate anatomy of the facial bones and complex vital structures surrounding them makes it challenging for the surgical teams to perform complex surgeries. With the rapid change in technology and modern advancement in virtual surgeries, there is a leap towards improvement in healthcare. To study biomechanical properties, it is imperative to include the principles of physical science in the field of medicine. In recent times, Finite element analysis (FEA) has become a useful tool to study the biomechanical properties of craniofacial structures under different mechanical parameters. Since the human structure's biomechanics is not possible to study on an experimental basis, finite element analysis has become an emerging tool to solve these complex biomechanical equations. The finite element method uses a numerical calculation of small heterogeneous geometry into the simple linear equation and predicts biomechanical responses towards each variation. Although used extensively in engineering, this method finds extensive use in the medical field, from planning surgeries to design external prosthesis. This method's most significant advantage includes studying a model outside the body, designing an idle surgical instrument and hardware, models that can be replicated based on user requirements, no ethical consideration needed, and print prosthesis that exactly resembles a typical anatomical structure. This method has certain limitations: high cost, technical flaws, and inability to replicate exact clinical conditions. This review article covers the current FEA scope in maxillofacial surgeries, steps in planning surgeries, advantages, disadvantages and the modifications needed to refine it for future research.
Corresponding Author: Dr. Manish Anand, Department of Oral and Maxillofacial Surgery, Meenakshi Ammal Dental College,

Chennai, Tamilnadu, India.

E-mail: manishanand028@gmail.com

DOI: $10.14260 / \mathrm{jemds} / 2021 / 414$

How to Cite This Article: Anand M, Panwar S, Bisht S. Role of finite element analysis in oral and maxillofacial surgery - a literature review. $J$ Evolution Med Dent Sci 2021;10(27):2024-2028, DOI: 10.14260/jemds/2021/414

Submission 14-01-2021, Peer Review 04-05-2021, Acceptance 10-05-2021, Published 05-07-2021.

Copyright () 2021 Manish Anand et al. This is an open access article distributed under Creative Commons Attribution License [Attribution 4.0 International (CC BY 4.0)]

\section{KEYWORDS}

Finite Element Analysis, Craniomaxillofacial Trauma, Orthognathic Surgery, FEA 


\section{BACKGROUND}

Oral and Maxillofacial Surgery is a specialized dentistry branch that deals with broad spectrum of diseases, injuries and defects in the head and neck region. Precisely describing this branch focuses on four significant areas - Minor oral surgery, Craniomaxillofacial trauma, Craniomaxillofacial deformities and head and neck pathology. With recent advancement in patient care, training and instruments, surgeons could bridge this gap to a specific limit. However, in modern science, it has become imperative to incorporate recent physics principles into the branch of medicine.

Finite element analysis (FEA) is one such branch of physics developed by engineers in different industries to address structural mechanical problems related to aerospace and civil engineering. ${ }^{1}$ This method incorporates calculation at different small discrete elements and combines to give a final result through numerical analysis. ${ }^{2}$ A user may modify the final result to interpret biomechanical behaviour and optimize the final surgical plan. ${ }^{3}$

The finite element method (FEM) has recently become an advanced analysis instrument to understand physical and mechanical interaction on an elemental level. It is the simulation of any given physical phenomenon using the numerical technique called Finite Element Method (FEM). Engineers use it to reduce the number of physical prototypes and experiments and optimize components in their design phase to develop better products, faster.

Several research teams have used the finite element method to know biomechanical properties and stress distribution in the maxillofacial region in recent years. For example, Antic $S$ et al. and salvific I et al. studied the influence of an unerupted lower third molar as a risk of mandibular angle and condylar fractures. ${ }^{4}$ Atac $\mathrm{M}$ et al. and Erkmen $\mathrm{E}$ et al. compared two versus four plate fixation using FEA in maxilla following Le Fort 1 osteotomy. Yeliz Kilınç et al. studied the mandibular angle's fragility because of third molars after trauma to the mandibular body. Yun - Feng LIU et al. studied stress distribution to mandible with various orientations of third molars. ${ }^{5}$

Finite element analysis has a wide area of scope in maxillofacial surgeries, including grafting of dental implant and understanding bone response, designing various titanium implant for reconstruction, designing surgical instruments, and studying the fracture line calculation of forces on the different anatomical area during major surgeries. This article will cover a brief overview of the application of FEA method mainly on four areas of interest, i.e. minor oral surgery, craniofacial deformity, craniofacial trauma and maxillofacial pathology.

\section{METHODS}

This review article summarises the conclusive shreds of evidence of the subject of finite element application in oral and maxillofacial surgery. The evidence was collected using search engine PubMed, Google Scholar and Medline. The keywords "finite element study", "finite element analysis", "oral and maxillofacial surgery", "craniomaxillofacial trauma", "orthognathic surgery" and combinations of keywords as mentioned earlier were selected from the databases. The retrieved articles were segregated into a group of different applications in the oral and maxillofacial surgery. The only study that falls in the range of level 1 - 3 of a hierarchy of evidence was chosen to provide readers with substantial knowledge of this subject. In the following subsections, the application of finite element analysis in various oral and maxillofacial surgeries is discussed briefly.

\section{Steps in Finite Element Analysis}

The process of FEA consists of 3 necessary steps.

\section{Construction of a Patient-Specific Virtual Model}

Patient-specific model is created from a digital model of a CT scan using a DICOM file. Additionally, for a non-living tissue Laser Scan can be used. These images are then converted into IGES and then processed through MIMICS or ANSYS software.3,6 These software have been designed to reproduce the $3 \mathrm{~d}$ model from imaging data accurately. To make a FEA model as accurate as a biological model, we need to assign material properties that closely resemble biological tissue. In this scenario, bone is primarily considered a solid material with Young's modulus of elasticity, Poisson's ratio, and density. With a series of experiments, scientists have concluded the specific value for the properties as mentioned earlier, and these values are assigned in a Finite element model to increase the accuracy of finite element study.

\section{Finite Element Generation and Assigning Boundary Condition} Once the 3D model is created, the next step is defining geometry to specify the interest domain (Figure 1). The domain of interest is divided into small segments called mesh. A mesh is a discretization of a domain existing in one, two or three dimensions. Using Materialise 3 - Matic, the mesh can be generated for further FEA analysis. 7,5

Each mesh contains a different number of interconnected nodes, and by increasing the number of nodes, one can increase the accuracy of the model. According to the literature, in most studies, researchers have used ten node 3-d tetrahedral quadratic elements. The distance between the nodes is also a critical criterion that determines the prepared model's absolute accuracy, and usually, the average distance between the nodes range from $0.25 \mathrm{~mm}-0.75 \mathrm{~mm}$.

Boundary conditions for a finite element model can be described as isolation of a study-specific area from the adjoining structures and restrict its movement in all three degrees of freedom so that the interference of movement is avoided during the application of force. For example, in mandible, condyles have translatory and rotatory movements during the mouth opening and closing. It is essential to restrict these motions as movement will hinder the final analysis. To make mandible function as a single unit, condyle heads are fixed in all three-planes with an application of spring, which is modelled as a muscle with no resistance during compression.

\section{Deriving Equation and the Post-Processing Phase}

The generated model acts as Master model on which the Finite element study is done; usually, a blunt trauma over a specified region is simulated. ${ }^{4}$ The transmission of force on every discrete particle is derived from a differential equation and is processed in a colour-coded chart (Figure 2). The graph has a 
spectrum ranging from red to blue. Red represents the area of maximum tensile stress, and blue represents the area of maximum compressive stress. Von Mises stress and maximum principal strain value can be calculated based on these values. These values determine whether the material will yield or fracture in a given clinical scenario. A solver is used in a postprocessing phase to solve the complex equation by undertaking physical information (impact force, muscular force, occlusal force, etc.) and solving equation digitally to derive result in a processed model.

\section{Finite Element Analysis in Craniomaxillofacial Trauma}

Facial trauma can cause significant morbidity to the individuals both in terms of aesthetic and functional. A surgeon should have a thorough knowledge of fracture line propagation's primary mechanism as it will help in precise surgical planning. Although based on patient history and study on the animal model in the past, clinicians were able to understand the mechanism to a certain extent. However, the outcomes were highly variable, and there was no substantial evidence to support their hypothesis. Because of the ethical, legal consideration, these studies and experiments were not possible on live human beings, so the finer details were often missed. With the advent of Finite element technology, clinicians can better understand the complex biomechanical behaviour of bones, fracture line propagation, mechanism of impact, and finally formulating an optimum surgical plan. ${ }^{8}$

FEA in craniomaxillofacial trauma relies on two basic principles - a line of fracture propagation and designing the patient-specific prosthesis to get ideal osteosynthesis along the least resistance line. Often, impact at the primary site causes fracture of the distant bone, commonly referred to as a countercoup nature of injury. ${ }^{4}$ With Finite element, the impact force is simulated on a virtual model and through numerical analysis, stress and strain pattern is studied. Also, the clinician may be able to know the quantum of force needed to induce fracture at a different site. This concept can be correlated clinically, and a judgement can be made between the nature of the impact and trauma pattern. In fracture reduction, we often immobilize the bony segments with biocompatible hardware like titanium plate, stainless steel plate, etc., which gives rigid strength to fracture segment until complete healing. Although precisely and rigidly designed, these implants can function to an optimum level but being a rigid device it acts as a source of stress generator. ${ }^{9}$ To overcome this, a manufacturer should exactly know the threshold value above which the implant can yield. This can be easily calculated by using FEA software, and the geometry of the plate can be designed, that can yield the muscular forces and dissipate extra load from the healing bone segments. ${ }^{10}$ Recently, clinicians have started designing different types of implants, configuring their geometry, and comparing them with a traditional implant to design an ideal implant that can be biologically and mechanically superior to available implants in the market.

\section{Finite Element Analysis and Reconstructive Surgeries}

Reconstruction of the defects secondary to the oncological resection is quite challenging for the surgical team. The aberrant anatomy of the maxillofacial region, characteristic of the defect, and aesthetic concern altogether make reconstructive procedures highly complex with limited options. With recent advancement, there is a paradigm shift from an autogenous mode of reconstruction to a custom-made prosthesis to avoid the complication of donor site morbidity. An ideal reconstructive material should accurately fit the defect size, withstand functional load and have biocompatible properties to integrate with the surrounding tissue. As of present, the surgeons favor titanium reconstruction plates as it fits into the criteria of ideal reconstructive material. Earlier titanium plates were designed based on anatomical configuration, but it lacks precision and stability as they were not patient-specific and defects specifically.

With the advent of Finite element technology, the surgical team can plan modelling of defect based on CT images and design prosthesis, which are both patient - specific and defect specific. Moreover, FEA also allows the surgical team to analyse the designed implant's biomechanical behavior and study the stress distribution over the fixation material and bone. The procedure involves the generation of the $3 \mathrm{~d}$ model from the CT images and segregating the tumour part. Once the tumour has been removed virtually, including safe margins, the obtained defect is mirrored to generate a patient-specific implant. ${ }^{4}$ After that, suitable material properties like the young's modulus and poisons ratio are assigned with necessary boundary conditions. Using ANSYS and 3 - MATIC software, an idle mesh is generated onto which Finite element study is conducted. FEA is a powerful tool that allows the researcher to study the broad component of a given material's mechanical properties. With the knowledge of the functional load and the trajectory of force distribution across the tissue, scientists can design implant, which is superior to the traditional implant available in the market. One of the main drawbacks of the commercially available implant is the fatigue fracture because of the over-bending and lack of passive fitting intra-operatively, which hampers the final surgical outcome to a great extent. Pre-operative designing of the implant using FEA improves the surgical accuracy and shortens the treatment period. Besides, the custom-made prosthesis can be made porous with an optimum strength that allows the bone tissues' growth through these pores.

Reviewing the literature, a study by Moiduddin et al. where he designed a customized porous reconstruction plate for the reconstruction of mandible secondary to the resection of plexiform-predominant ameloblastoma, claimed that the designed implant was resistant to the occlusal forces of the muscles of the mastication and provided good stability for the reconstructed mandible. Gutwald et al. have a similar finding in his study. He analysed seventy-six different plate design types for mandibular reconstruction and concluded that customised reconstruction plates have a better biomechanical performance than manually bent stock reconstruction plates in terms of statistically significant fatigue limit. An interesting study by Atilgan et al., where he investigated the possible reasons for breaking the titanium reconstruction plates using finite element analysis, concluded that by modifying the weak stress concentrating area, one could modify the reconstruction plate for a better outcome based on the findings observed in the study. Although the customised reconstruction plates are more expensive than the standard plates considering the reduction in operating time by $30-40 \%$ and superior 
outcome, it is worthwhile to incorporate FEA technology in all craniofacial reconstructive surgeries.

\section{Finite Element Analysis in Minor Oral Surgery}

One of the minor oral surgery elements' main applications is to study dental implant behaviour and design an implant that can yield masticatory load. A key factor for the success or failure of a dental implant depends on the propagation of stress transferred to the surrounding bone. FEA gives researchers leverage to study stress pattern in the implants' interface area with cortical bone and around the surrounding of the implants in medullary bone. ${ }^{11}$

Determining force distribution at the bone-implant interface is an integral step in the overall loading survey, which determines an implant's fate. It has long been hypothesised that both implant and bone should be in equilibrium within a specific load range for physiological homeostasis. ${ }^{12}$ Undue stresses can cause bone resorption or fatigue failure of the implant, whereas underloading the bone may lead to disuse atrophy and subsequent bone loss. ${ }^{12}$

Load transmission and resultant stress distribution at the bone-implant interface have been the area of interest of FEA studies. $^{13}$ Factors that influence load sharing at the boneimplant interface include the type of loading, implant and prosthesis material properties, implant length and diameter, implant shape, the structure of the implant surface, nature of the bone-implant interface, and the quality and quantity of the surrounding bone. 11 of all these biomechanical factors, implant length, diameter, and shape can be modified easily in the implant design, and with the incorporation of FEA analogy, these properties can refine near to idle. ${ }^{13}$

\section{Finite Element Analysis and Craniofacial Deformity}

Craniofacial deformities are a diverse group of deformities in the growth of the head and facial bones. It can present as a congenital or acquired secondary to any trauma or surgical resection. Understanding the growth pattern and intervening early can help the clinician get an optimal result. Earlier based on the serial radiographs, growth pattern was studied, but radiograph has its superimposition limitation. The FEA method has been applied to study growth pattern at a subsequent level to eliminate such a disadvantage. Moss et al. studied the pattern of craniofacial growth in rats using FEM, where the craniofacial skeleton of the rat was integrated into two-dimensional tri-angular elements. ${ }^{14}$

They concluded that the craniofacial skeleton's growth could be evaluated more quantitatively because FEM growth analysis is independent of any geometric constraints related to a reference frame or superimposition method employed in radiographs and proposed the usefulness for growth analysis for the first time. Based on this concept, a craniofacial model is discretized into several small elements, and for each element, stress and strain values were calculated. The obtained values were then compared with the usual subjects. Recently finite element technology is being used to study the pattern of growth in cleft lip and palate patients following nasoalveolar molding (NAM) therapy. In craniofacial literature, NAM therapy has always been a matter of debate because of the poor understanding and lack of evidence of NAM therapy's growth pattern. Perhaps, the study by Ritschl et al. can be taken as the most valid evidence to support the feasibility of NAM therapy. In this study, the author analysed the stress distribution pattern in neonates during NAM and concluded that Nasoalveolar molding therapy is a safe presurgical treatment option without a remarkable impact on the viscero neurocranium of neonates. Another significant advancement in the field of craniofacial surgery is the designing of the distraction device, used for incremental bone growth under controlled traction. The distractor works on the principle of resultant vectorial force which is simulated using the mechanical appliance. FEA has been widely applied to design distraction devices that are more stable, accumulate less stress and induces positive mechanical forces to aid in the bone growth.

\section{Finite Element Analysis in Orthognathic Surgeries}

One of the essential factors determining the success of orthognathic surgery is the post-operative stability of bridging the bony segments with various fixation systems. FEA has been extensively applied to study stress distribution across this fixation system on a 3 - $d$ model, to know material properties that will effectively contribute to designing ideal fixation system, which will be more superior and biocompatible with the system available now. Another, meaningful application of FEA in orthognathic surgery is understanding the pattern of split or disjunction after osteotomy. ${ }^{6}$ For example, pterygomaxillary disjunction in Le Fort surgery is considered as the most critical and complex step intra-operatively, determining the surgery outcome.15 FEA has been introduced to study the pattern of a split by mechanically stimulating the 3-d model and knowing the quantum of force generated during these splits. ${ }^{16}$ It gives surgeons an idea of the path of split and designs an instrument that will be biomechanically more stable to minimize unfavourable stresses on neighbouring anatomical structures.

\section{Limitations and Future outlook}

In a nutshell, FEA helps the researcher study complex parameters individually and have the window of repeating experiments as often as possible, which would have been impossible with other in-vitro studies. However, FEA studies' significant drawback is a generalisation of a parameter value assigned during the study. The value assigned is based on material properties and is considered to be uniform for every subject. For example, Young's modulus for cortical bone has been mentioned in a range of $13,500 \mathrm{Mpa}$ to $18000 \mathrm{Mpa}$ in literature, which will not be same for every patient and hence it will create bias in the study. ${ }^{6}$ Another issue is replicating clinical conditions into the FEA model since the conditions are programmed by computer, there is always a chance of missing on the complex anatomical properties. ${ }^{7}$ Moreover, differentiation and quantifying hard and soft tissues are quite challenging because FEA studies are based on the isotropic model, and the heterogenicity of living tissue makes it complex to determine the qualitative result.

Future research intends to modify the FEA model, which will be clinically more accurate and solve more complex problems. At present, scientists are trying to create finer 
meshes of finite element model by increasing the number of elements and nodes. There is also a consensus for mesh-less analysis which will reduce the meshing time, more accuracy and demand of fewer numbers of the degree of freedom. Another advancement is calculating the young's modulus from bone density by modifying the CT measurement unit to help the researcher identify the weaker and stronger region of the maxillofacial skeleton to create a more realistic mode. ${ }^{11,4}$ The non-linear property of the material like friction and sliding can remarkably affect the principal strain and stress created on the contact surfaces. FEA's technical limit could be possibly solved by understanding unpredictable parameters like region of contact, temperature, vectorial load, and humidity.

\section{CONCLUSIONS}

To conclude, FEA is a powerful tool that has got a unique role in maxillofacial surgery. Many of the earlier hypotheses that were only theoretically proven are now being backed up by mathematical and geometrical equations using FEA. In addition to this, FEA technology has been extensively useful by surgical instruments manufacturers as it provides data that helps an engineer to design more sophisticated and stable instruments. On the contrary, FEA's technical limits could be possibly resolved by a strong collaboration of engineering and medical society. With a better understanding of physics and its implication, maxillofacial surgeries will ultimately become an evidence-based practice in the near future.

Financial or other competing interests: None.

Disclosure forms provided by the authors are available with the full text of this article at jemds.com.

\section{REFERENCES}

[1] Szücs A, Bujtár P, Sándor GKB, et al. Finite element analysis of the human mandible to assess the effect of removing an impacted third molar. J Can Dent Assoc 2010;76(1):72.

[2] Bezerra TP, Junior FIS, Scarparo HC, et al. Do erupted third molars weaken the mandibular angle after trauma to the chin region? A 3D finite element study. Int J Oral Maxillofac Surg 2013;42(4):474-80.

[3] Gröning F, Liu J, Fagan MJ, et al. Validating a voxel-based finite element model of a human mandible using digital speckle pattern interferometry. Journal of Biomechanics 2009;42(9):1224-9.
[4] Antic S, Vukicevic AM, Milasinovic M, et al. Impact of the lower third molar presence and position on the fragility of mandibular angle and condyle: a three-dimensional finite element study. J Craniomaxillofac Surg 2015;43(6):870-8.

[5] Liu YF, Wang R, Baur DA, et al. A finite element analysis of the stress distribution to the mandible from impact forces with various orientations of third molars. J Zhejiang Univ Sci B 2018;19(1):38-48.

[6] Lisiak-Myszke M, Marciniak D, Bieliński M, et al. Application of finite element analysis in oral and maxillofacial surgery-a literature review. Materials (Basel) 2020;13(14):3063.

[7] Trivedi S. Finite element analysis: a boon to dentistry. J Oral Biol Craniofac Res 2014;4(3):200-3.

[8] De Mello Santos LS, Rossi AC, Freire AR, et al. Finiteelement analysis of 3 situations of trauma in the human edentulous mandible. J Oral Maxillofac Surg 2015;73(4):683-91.

[9] Aquilina P, Parr WCH, Chamoli U, et al. Finite element analysis of patient-specific condyle fracture plates: a preliminary study. Craniomaxillofac Trauma Reconstr 2015;8(2):111-6.

[10] McHenry CR, Wroe S, Clausen PD, et al. Supermodeled sabercat, predatory behavior in smilodon fatalis revealed by high-resolution 3D computer simulation. Proc Natl Acad Sci U S A 2007;104(41):16010-5.

[11] Özgen C. Finite element invesitgation of mechanical interaction of dental implants with bone. Doctoral dissertation, Middle East Technical University, 2007.

[12] Cicciù $M$, Cervino G, Milone $D$, et al. FEM investigation of the stress distribution over mandibular bone due to screwed overdenture positioned on dental implants. Materials (Basel) 2018;11(9):1512.

[13] Sripriya S. Evaluation of thread design of conventional implant and indigenous implant in implant retained auricular prosthesis: in vitro study. The Tamil Nadu Dr. M.G.R. Medical University, 2010.

[14] Moss ML, Skalak R, Patel H, et al. Finite element method modeling of craniofacial growth. Am J Orthod 1985;87(6):453-72.

[15] Fujii H, Kuroyanagi N, Kanazawa T, et al. Threedimensional finite element model to predict patterns of pterygomaxillary dysjunction during Le Fort I osteotomy. Int J Oral Maxillofac Surg 2017;46(5):564-71.

[16] Larson BE, Lee NK, Jang MJ, et al. Comparative evaluation of the sliding plate technique for fixation of a sagittal split ramus osteotomy: finite element analysis. Oral Surg Oral Med Oral Pathol Oral Radiol 2017;123(5):e148-52. 